\title{
Blockverse: A Cloud Blockchain-based Platform for Tracking in Affiliate Systems
}

\author{
A. Baldominos ${ }^{1,2 *}$, J. L. López-Sánchez², M. Acevedo-Aguilar ${ }^{2}$ \\ ${ }^{1}$ Computer Science Department, Universidad Carlos III de Madrid (Spain) \\ ${ }^{2}$ Blockverse Solutions S.L., Madrid (Spain)
}

Received 6 April 2020 | Accepted 14 April 2020 | Published 15 June 2020

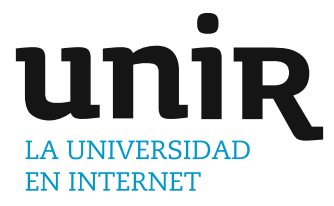

\section{ABSTRACT}

\section{KEYWORDS}

Affiliate systems are a crucial piece of today's online advertising. In affiliate systems, web traffic is directed from certain sites displaying ads to the websites of those company whose products or services are advertised. The way in which these ads are monetized is diverse and can respond to different models. In many cases, affiliates establish a cost based on impressions (displays of the ad) or on clicks. However, more intricate models are becoming widespread, such as the cost per action, where the affiliate incomes are due to the users performing certain actions in the target website. In particular, in the world of iGaming, it is frequent that affiliates charges are based on registrations, deposits or money lost on bets. In this scenario, Blockverse is a tool whose objective is to record transactions occurring in affiliate systems at large scale, using a permissioned blockchain implemented atop state-of-the-art cloud technology. Additionally, the system will be able to execute smart deals that generate income for affiliates based on the agreed conditions, and to provide real-time analytics in the context of the affiliate system.

\author{
Blockchain, Cloud \\ Computing, Databases, \\ Affiliate Systems, \\ Analytics.
}

\section{INTRODUCTION}

$\mathrm{A}^{\mathrm{H}}$ FFILIATE systems play a key role in today's online advertising scheme, as their presence is key to gain web traffic to those websites whose products or services are being advertised.

Although the methods and agreements by which affiliate systems are governed are quite diverse and strongly depend on the industry whose products or services are advertised, the idea behind all of them is quite simple: affiliate systems sell the product of another company (the advertiser) at an agreed fee or commission.

Now, this sale can take place in different ways. In this paper, we will focus in a certain type of affiliate systems which direct web traffic to the advertiser, with the latter performing the final sale. In other words, the sale itself is performed as usual, except for the fact that the customer did not arrive organically, but forwarded by another company, which must receive the agreed fee.

Again, the deals involving how these fees are determined can vary significantly between industries and products. One example of such fees can be found in the Amazon Associates affiliate program [1], where affiliates can sell products of Amazon.com for a certain commission. As of March 2020, this commission is determined as a percentage based on the category of products (e.g. 10\% in luxury and beauty, $4.5 \%$ in physical books, etc.) or as a fixed fee for the registration in some services (e.g. \$3 for registering into Amazon Prime free trial).

In other cases, commission fees are subject to specific agreements resulting from negotiations between the partners (the affiliate and the advertiser), that could be re-negotiated in the future.

\footnotetext{
* Corresponding author.

E-mail address: abaldomi@inf.uc3m.es
}

In this paper, we will put the focus on the industry of iGaming. In this setting, we will call operators to the advertisers, since they are expected to have a license to operate in the iGaming market. Examples of operators can be online casinos or bet platforms.

In iGaming, the deals governing how affiliates perceive money can be quite intricate and complex. For example, the affiliate may perceive a fixed fee if a customer registers in the operator's platform. Additional fees, either fixed or variable, can be agreed if the customer deposits money or losses it in a bet.

Additionally, there might exist several agreements established between an affiliate and an operator, based on how the customer arrived into the operator's platform. For example, certain advertising sites can be paid at a better rate or, in some cases, the fees can be determined based on the size of the ad, etc.

This complex scheme makes it critical to carry out secure tracking of customers, from the moment they arrive at the advertising to their steps in the operator's platform. The identification of customers must be unequivocal in order for affiliates to perceive agreed fees correctly.

In this scenario, it is frequent that a certain degree of distrust arises. In many cases, a chain of intermediaries exists between the affiliate and the operator in order to alleviate some of this distrust, therefore adding extra costs to the entire advertising chain.

In a setting like this, a Blockchain-based system can serve as a technical solution for securely and trustily logging the interactions and communications between affiliates and operators, without the need for additional intermediaries, therefore reducing costs while keeping trust.

The Blockchain would be used as an immutable ledger storing all of the transactions taking place in the advertising scheme, therefore enabling the partners to performed advanced analytics, while at the same time would allow the establishment of custom smart deals 
between affiliates and operators in order to govern how exchanges of traffic and money are carried out.

In this paper we are devising Blockverse, an online platform based on Blockchain for tracking the transactions between affiliates and operators in the iGaming industry. In particular, the paper explains technical decisions made for allowing Blockverse to scale and to provide a solution to the problem of tracking customers, traffic and transactions.

The paper is structured as follows: section II presents some basic concepts and notation that will be useful to understand the remainder of the proposal, aimed at people not familiarized with the industry or with affiliate platforms. Later, section III presents the current state of the art, summarizing related works where the Blockchain is being studied as a solution for similar problems and domains.

The Blockverse platform is described in section IV, illustrating its user experience and discussing how communication between affiliates and operators is established. This is followed in section $\mathrm{V}$ by a technical discussion of how the system is designed to scale to large amounts of traffic. Finally, section VI presents some conclusive remarks of this paper and poses interesting lines for future research.

\section{BASIC CONCEPTS AND TERMINOLOGY}

In this section, we introduce some basic notation that will be useful to understand the remainder of the proposal.

\section{A. Concepts Related to Affiliates}

An affiliate is an entity that forwards traffic to the advertiser, which in the iGaming industry we also refer to as the operator. The affiliate is interested in that this traffic is of quality, in the sense that it leads to a higher rate of conversions (specific actions in the operator's platform), since this will often result in higher revenues.

The following concepts are closely related to the role of the affiliate in the advertising scheme:

- Money site: it is a webpage designed to redirect traffic to the operator's platform. This webpage might contain ads in the form of banners, videos or other form of sponsored content with links to the operator's site.

- Asset: it is a resource which is placed in money sites and serves for the purpose of forwarding the customer to the operator's platform, such as a clickable banner.

- Click event: it is an event initiated by the customer (an online users) that results into a redirection of traffic to the operator's platform, such as clicking an asset.

\section{B. Concepts Related to Operators}

An operator is a company which holds a license to operate in the iGaming industry, such as a casino or betting platform. Due to the nature of this industry, there are strong regulatory platforms to ensure that companies provide a reliable service to customers, and companies must adhere to these regulations to operate.

The following concepts are closely related to the role of operators in the advertising scheme:

- Platform landing page: it is the website in which a customer lands after performing a click event. This website will often present the operator's features and allows the customer to register, in some cases providing customers with some offers or other advantages for promoting their enrolment.

- Brand: in some cases, operators might present to their customers different brands or commercial names. For example, a single operator might have one brand for an online casino and a different one for sports bets.
- Register event: it is an event initiated by customers that results in their enrolment in the platform.

- Deposit event: it is an event initiated by customers that leads to the deposit in their platform's account of a certain amount of money. This money can later be used for betting and can be incremented or decremented based on the outcome of bets. This amount can also be decreased when customers transfer money to their bank account.

- Bet event: it is an event initiated by customers that consist in betting a certain amount of previously deposited money. This bet can take place in an online roulette, a poker game, a soccer match, etc.

- Win/lose: it is the outcome of a bet event. This outcome might be known immediately after the bet (for example, in an online roulette) or after some time (for example, when a soccer match has concluded). A win outcome means that the customer wins some money beyond the betted amount, therefore increasing the money in the deposit. A lose outcome means that the customer loses money, which might be all of the betted amount. It is worth noticing that the operator earns money when the customer loses a bet.

\section{Users vs. Customers}

So far, we have been using the term customer to refer to an online user during the whole interaction process, including when he/she visits a money site. This terminology might be imprecise and lead to confusion, since a user might not be considered as such before registration in the operator's platform, or even before deposit.

Properly speaking, a user is not considered a customer until a certain event occurs, which is known as the conversion. Most often, a user that lands into the operator's platform (e.g. after a click event) is not yet considered a customer, although has potential to become into one.

In some cases, the conversion event is the register event itself. In other cases, a user might not be considered to convert into a customer until a deposit takes place. This can happen, for instance, in some platforms with a small ratio of deposits/registrations.

Anyhow, the difference between users and customers is not a critical issue in this paper. Later, we will see how the Blockverse platform has chosen a term to denote both users and customers regardless of when conversion takes place.

\section{Concepts Related to the Market}

Besides those concepts that are exclusive to affiliates or operators, there are some important terms that are relevant in the interaction that arises between them:

- Partnership: it is the agreement between an affiliate and an operator to establish certain deals for the former to redirect traffic to the latter.

- Transaction: it is any exchange of information between the two partners. Additionally, a transaction can also take place between the customer and the operator, but even in this case the affiliate will be considered a third-party in the transaction.

- Deal: it is an agreement of conditions for the affiliate to perceive some revenue due to customers generated by their traffic. These conditions can be very complex and can depend on aspects such as the money site, the asset generating the traffic, etc.

In section IV we will provide more specific details on the different types of deals that are supported in the Blockverse platform.

\section{STATE OF THE ART}

The blockchain is a concept generally acknowledged to arise in the original white paper describing the Bitcoin electronic currency by 
Nakamoto [2]. It was originally developed as an auxiliary technology to support such cryptocurrency, providing the security mechanisms guaranteed by Bitcoin and enabling the mining procedure.

Later on, larger possibilities of the blockchain technology as Buterin described its ability for storing and running decentralized apps and smart contracts [3], which eventually led to the development of the Ethereum platform and the Ether cryptocurrency.

Since the mid-2010s, the blockchain has emerged as an interesting technology enabling a large variety of applications. A survey by Zheng et al. [4] in 2018 reveals a wide spectrum of applications to fields as diverse as finance, security and privacy, internet-of-things, reputation systems and public and social service. More recently, Baldominos et al. [5] have suggested mechanisms for evolving proof-of-work into mechanisms allowing for the distributed training of artificial intelligence applications.

An interesting feature of the blockchain is that it is often described as a mechanism for shedding trust into ecosystems where different parties interact with each other. For example, Blasingame [6] contributed an article to Forbes describing blockchain technologies as "the future of trust". Anjum et al. [7] described how blockchain could bring compliance and trust to different applications outside the scope of finance and banking. Also, Hawlitschek et al. [8] described how the blockchain is able to replace trust to a certain extent in the context of sharing economy applications.

In this paper, we put the focus into an application of blockchain to online advertising and affiliate systems. The literature coverage of this topic is rather narrow. A broad analysis of the impact of blockchain technologies on marketing was published by Ertemel in 2018 [9].

A more relevant work was presented by Pärssinen et al. [10], where they describe the potential of blockchain to online advertising. In this paper, authors enumerate some of the typical problems of the industry: large numbers of intermediaries adding costs and exploiting users' data, and fraud in the advertising chain. They end up concluding that further research is required to understand whether successful applications of blockchain to online advertising can be implemented. The issue of fraud has been further studied by Kshetri and Voas [11], suggesting that blockchain has a potential for tackling fraud, despite of some challenges regarding its application.

To the best of our knowledge, there are not published works describing applications of blockchain to affiliate systems. However, an early attempt was suggested by Weijer in his Master's thesis [12]. In his work, Weijer identifies some opportunities for blockchain in the scope of affiliate marketing and suggests a design for such a system, concluding that blockchain could improve transparency in the system.

Finally, although previous implementations of a system like this in the industry are not known by the authors to exist, a patent registered by IBM in 2016 [13] settles the foundations for a transactions tracking system bases on blockchain.

\section{The BlockVerse Platform}

In this paper, we are proposing and describing the implementation of a Blockchain-based platform for tracking the transactions taking place between affiliates and operators in an iGaming setting.

The rationale behind this platform is to enable a secure and immutable exchange of information between the partners, without the need of third-party intermediaries to preserve the trust of the system.

Behind the blockchain database used to store transactions, the platform is designed to enable an end-to-end interaction between the partners. This includes adding new partners to their network, establishing new deals, exploring transactions in real time, and checking out summary analytics that are automatically computed by the system. Therefore, Blockverse aims to be an integrated solution combining tracking of operations and enforcement of the execution of deals, as well as providing a business analytics tool.

This section provides a logic description of the key elements comprised in the Blockverse platform, whereas section $\mathrm{V}$ will delve into the technical details of the solution, putting the focus on the issue of scalability. Finally, section VI will provide some conclusive remarks about the Blockverse platform, and outline some lines of future work and improvements.

\section{A. The Blockchain}

The Blockchain is the database infrastructure used to store all the transactions taking place in Blockverse. In the most fundamental terms, a blockchain is no more than a chain of blocks, with each block storing certain information.

The blockchain technology; however, is famous for constituting the foundation of several well-known cryptocurrencies, such as Bitcoin or Ethereum. Decentralized blockchain networks such as those supporting these currencies provide mechanisms for ensuring integrity and security of the data via cryptographic mechanisms that take place during the process known as mining.

However, some of these cryptographic mechanisms can be used in a different type of blockchain network to still preserve data integrity. In particular, Blockverse relies on a centralized permissioned private network which implements security mechanisms to avoid manipulation of the ledger. These features of the blockchain technology underlying Blockverse have the following meaning:

- Centralized: the database is stored in a centralized system, as opposed to a distributed system composed of a network of nodes carrying out the mining process.

- Private: the database is not public, meaning that not anyone has access to its contents.

- Permissioned: the database will provide selective access of the contents of the blockchain to users based on their permissions, meaning that a given user will often have a very restricted view of the database (such as those transactions in which he/she is involved).

The blockchain technology used for Blockverse is the result of a custom implementation, which is strongly influenced by the Bitcoin technology. In this implementation, transactions are stored in the database and are periodically included into a block. Since the blockchain is centralized, a distributed mining system does not make sense. However, Blockverse has incorporated an automated mining system which confirms blocks after ensuring that all transactions located within them are valid and consistent with the rules governing the market.

As in Bitcoin, a cryptographic mechanism is implemented to ensure the integrity of the chain. In particular, each block is hashed using SHA3-512 and the hash of a block is used as an element of the following block (and included in the latter's hash). Therefore, trying to break the integrity of the blockchain will raise an alarm, as hashes would not match their expected values.

\section{B. Trackers}

In section II we explained the difference between a user and a customer. This distinction can be hard to do because it will often depend on the definition given by operators, when in some cases a customer will be a registered user and, in others, the user will need to deposit a certain amount to become a customer.

In any case, Blockverse has introduced the concept of tracker to allow the system to remain agnostic about the real nature of users and 


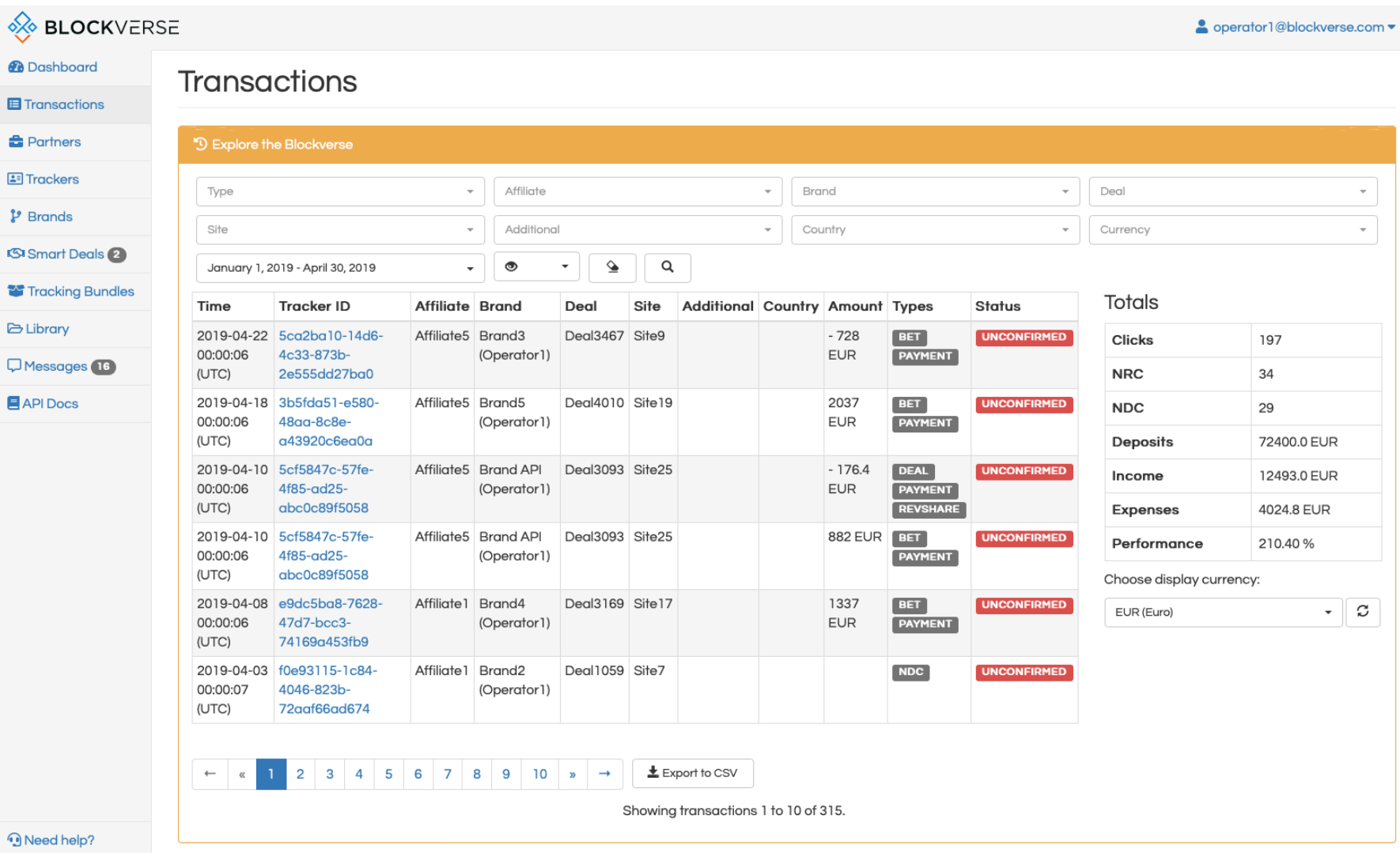

Fig. 1. Transactions screen in Blockverse. In this view, both affiliates and operators can see the list of transaction, applying filters as required. Custom fields are shown as columns in the table and are displayed based on the permissions of the logged profile. A section of totals is shown in the right side, providing some metrics of interest for the filtered data. All transactions can be exported to a CSV file, so affiliates and operators can perform more complex queries or data analysis processes as required.

customers. A tracker is an entity that needs to be tracked, regardless of whether the conversion takes place.

A tracker is generated in Blockverse as soon as a click event takes place. In that moment, the user is forwarded to the operator's platform and the unique identifier of the tracker freshly generated is provided to the operator as part of the redirection process. During the tracker creation, additional information might be stored about how this tracker emerged, for example, the money site and asset that led to the generation of the tracker, etc. This will be useful to choose which smart deals (specific business logic affecting the revenue of affiliates) will govern the actions performed by such tracker, if any.

After landing in the operator's platform, the operator will be in charge of linking the tracker identifier to any of the operations performed by the tracker, and particularly, reporting to Blockverse the register, deposit, bet and lose/win events. Such events will be used with two purposes: (1) triggering smart deals that generate revenue to affiliates based on the tracker actions and (2) providing accurate business analytics and key performance indicators to both affiliates and operators about the behavior of trackers.

Even if the user does not perform any additional action beyond landing in the operators' platform, Blockverse will create the tracker, since it can be used by affiliates to get a summary of the performance of their different money sites and assets.

Finally, trackers in Blockverse have an associated key-value data store, which can be used by partners to provide additional information about trackers. Interestingly, these data are stored outside of the blockchain; instead, the blockchain only stores a unique identifier of trackers in the transactions in which they are involved. This means that this data store can be used to fill trackers with information known about them (name, email, etc.) allowing GDPR compliance since the data could be modified or removed if requested by the user.

\section{Transactions}

In Blockverse, a transaction is any kind of interaction between partners that is stored in the blockchain. Most transactions will always involve three parties: an affiliate, an operator, and a tracker.

Transactions in Blockverse are labelled with a type and can have associated data in the form of a key-value store.

While the blockchain implementation available in Blockverse provides support for transactions of any type, the transaction types are currently restricted to the following: new (for click events), nrc (standing for new registered customer, it used for register events), deposit, $n d c$ (standing for new deposit customer, it is recorded the first time a tracker completes a deposit event), bet and payment. Affiliate platforms can record transactions of type new, whereas operators can record any of the other.

Payment transactions can have two different sources. On the one hand, they can be reported by the operator when a win/lose event takes place, indicating which are the source and the target of the payment.

On the other, pay transactions can be generated automatically by smart deals as a result of commission fees due to the events performed by the tracker. These pay events represent income of the affiliate that should be invoiced to the operator at the end of the period.

Regarding the data store associated with a transaction, it is able to store any kind of data. These data can be introduced either by the affiliate or by the operator when a transaction is created.

Data items associated with a transaction are permissioned as well, meaning that the owner of the transaction (the partner that records it) 
can decide whether a piece of data is private or public. By public, we mean that the other partner has access to it. As a result, private data can be useful for an affiliate or an operator to store information internal to their business logic or that is confidential.

An example of how transactions are displayed in the Blockverse website is displayed in Fig. 1. It can be seen how different filters can be applied and some summarizing metrics are computed for the specified period and conditions.

\section{Smart Deals}

Smart deals in Blockverse constitute a mechanism that mimics the idea of smart contracts that is present in some blockchains, such as Ethereum. Unlike smart contracts, Blockverse deals are not stored inthe blockchain, but are stored as rules in the application, that are checked for execution every time new transactions are recorded.

Smart deals are the technology enabling the computation of amounts of money that operators shall pay affiliates for their services. The contents of smart deals are negotiated by partners and are they are recorded as application logic once terms have been agreed.

As of 2020, four types of smart deals can be established:

- Flat fee: it allows to set up a monthly fee that is paid from operators to affiliates regardless of traffic. This is a special kind of smart deal, since it is not triggered by transactions recorded in the blockchain, but on a timely basis.

- CPA: it stands for cost per action and allows to pay a certain fixed amount when an action occur. At this moment, the only supported action is NDC; i.e., when a customer makes a first deposit of money in the operator's platform. In this case, partners should agree on the CPA amount (the amount that will be paid to the affiliate if the action takes place) and can also establish a minimum deposit threshold, meaning that the payment will only take place if the users deposit at least that amount of money in the operator's platform.

- Revenue share: it allows to pay a variable amount of money based on the operator's revenue coming from lost bets. This amount is agreed as a percentage.

- Hybrid: it is a combination of CPA and revenue share, allowing to define both a fixed amount based on NDC actions and a variable amount based on revenue.

When trackers are created, they are already linked to a certain smart deal of CPA, revenue share or hybrid type. The way in which deals are linked to trackers will be explained later in this section. This means that affiliates and operators can apply different deals based on the source of traffic; for example, certain privileged positions (e.g. larger ads) can be subject to more interesting deals for the affiliate.

For all of these deals, partners can agree on a time threshold (specified as days, weeks or months), during which the deal will remain active for each tracker. This allows operators to decide for how long they will be paying affiliates for the revenue of a tracker or, in the case of CPA deals, after how much time they will not pay the affiliate for a new deposit. In either case, the period of time starts counting on the creation of the tracker.

Additionally, deals can be set up with an expiration date. This value is tracker-agnostic, meaning that after that date the deal will no longer be triggered, regardless of how many trackers are associated to it.

Finally, Blockverse provides an interface to create and negotiate deals. Smart deals can be created by either affiliates or operators, and they have to choose the partner to whom the deal will be established. After creation, the deal's status is "awaiting approval", and the recipient partner will be notified that a new deal is pending for verification. The screen available in Blockverse for creating a new smart deal is shown in Fig. 2.

\section{ST Create smart deal:}

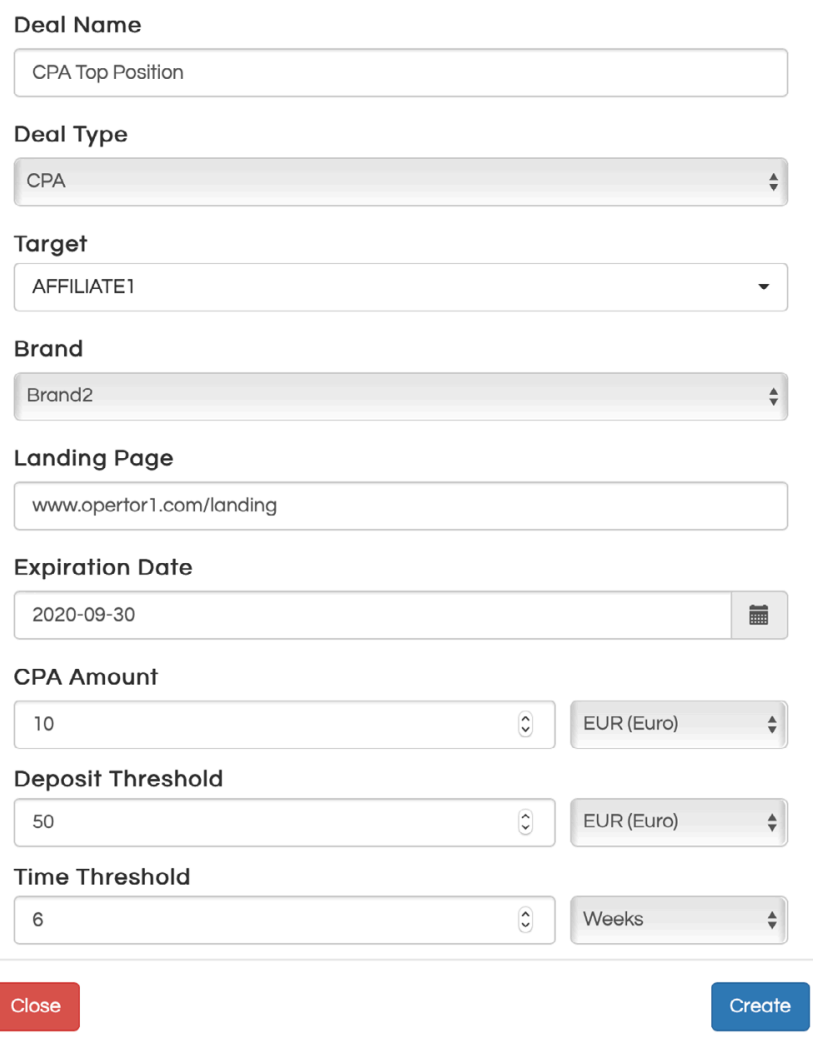

Fig. 2. Screen for setting up a new smart deal in Blockverse. In this example, the view for a new CPA deal is shown, although logic is similar for flat fee and revenue share deals. Once the deal is created, the partner must approve it.

At this moment, the recipient partner can decide to accept the deal, deny it or make further modifications to it. If the deal is approved, it will be set up as "active" and will start working. If the deal is "denied", it will be erased from the platform, and the issuer will be notified. If the deal is modified, it is returned to the issuer for its approval.

Active deals can be modified or cancelled, but in any of the cases, a request is issued to the other partner, and changes are not applied in the deal's logic unless the other party accepts the changes. This process ensures that deals are never modified unilaterally.

\section{E. API and Tracking Bundles}

Blockverse allows both affiliates and operators to record transactions using a RESTful HTTP API. All of the business logic, including the workflow of smart deals and retrieval of analytics, can be handled through this API.

A typical use case would be the following: an affiliate and an operator agree on a smart deal (either using the Blockverse interface or the API). Once the deal is created, it can be referred to using its unique identifier. The affiliate will set up an ad in a money site of its property, that will redirect traffic to the operator.

When the ad is clicked, the affiliate will call the Blockverse API to create a new tracker linked to the previously created deal. The Blockverse API will return a unique identifier for the tracker, and the affiliate will provide the operator with this identifier. In subsequent actions of the tracker, the operator will call the Blockverse API to record such transactions, communicating the tracker identifier. During each transaction, Blockverse will automatically check if the smart deal associated with the tracker involved in the transaction should be triggered, doing so if required. 
To ease this whole process, Blockverse also provides a mechanism known as "tracking bundles", which is a method to simplify tracker creation on the side of the affiliate.

When creating a tracking bundle, the affiliate will need to specify the operator and operator's brand, the smart deal involved and the landing page on the operator's platform. Moreover, the affiliate can set up custom fields in the form of pairs of keys and values, that will be stored along with the transactions resulting from the interaction with that tracking bundle.

Operators can also see tracking bundles referred to them and add additional custom fields. In either case, these fields visibility can be adjusted as public or private, and in the latter case, only the owner will be able to see them. The interface for creating new tracking bundles in Blockverse can be found in Fig. 3.

\section{Create new tracking bundle:}

\begin{tabular}{l} 
Name: \\
TrackingBundle \\
Operator: \\
Operator1 \\
Brand: \\
No brand \\
Deal: \\
\hline CPA Top Position \\
Redirect: \\
\hline www.operator1.com/landing \\
Tracking Fields: \\
\hline Site \\
\hline Position : TopCarousel \\
\hline + New Tracking Field \\
\hline Close
\end{tabular}

Fig. 3. Screen for setting up a new tracking bundle. Both affiliates and operators can set up custom fields and adjust their visibility.

After creating a tracking bundle, Blockverse will provide an URL that can be called directly by the affiliate when the click event takes place (for example, as the target URL of an ad). When this URL is called, Blockverse will automatically create the tracker and redirect the user to the operator's platform (in particular, to the URL specified in the "Redirect URL" field in the bundle creation form, see Fig. 3). The identifier of the freshly created tracker will be passed to this URL as a query parameter.

Additionally, the Blockverse platform will also display cURL and Python code required to record the click transaction with the data specified in the tracking bundle, to ease API integration.

\section{F. Revokation of Transactions}

Since all the transactions recorded in Blockverse are stored in a blockchain, past transactions cannot be deleted, even if they are known to be wrong (e.g., an operator records a transaction incorrectly).

To tackle this problem, Blockverse enables a mechanism to flag transactions as "revoked" that do not break the blockchain integrity.
This is carried out using a separate list of pointers to those transactions that are revoked.

Additionally, operators are given the possibility to revoke trackers. This mechanism is implemented to prevent potential fraud; e.g., affiliates redirecting traffic using a different deal than the one agreed to obtain higher revenues. By revoking a tracker, all past and future transactions associated to it are revoked, including those that constitute a source of revenue for the affiliate. New transactions will be still recorded, although they will be automatically revoked, and smart deals will no longer be executed.

\section{G. Dashboard and Business Analytics}

So far, we have described the features of Blockverse regarding the logic by which transactions are recorded and smart deals are executed.

Beyond this functionality, Blockverse provides a dashboard that displays relevant information to both affiliates and operators, that can be used to better understand their sources of traffic and revenue and take direct actions accordingly.

The top part of the dashboard provides the following information:

- Total clicks in the current month.

- Total NRCs (new registered customers) in the current month.

- Total NDCs (new deposit customers) in the current month.

- Total deposits in the current month.

- Total revenue in the current month.

All of these data are provided along with the information for those metrics in the same period of the previous month, allowing partners to better understand their current performance.

Additionally, some graphics are provided to display in a single image some relevant information. In the case of affiliates, the following graphics are displayed:

- A cumulative bar chart showing total revenue by position. Only the top ten positions are shown, and revenue is split based on whether it comes from CPA or revenue share.

- A monthly timeline plotting the evolution of the clicks to NRC ratio for the top five operators. This allows affiliates to know which operators are converting more traffic into registered users.

- A monthly timeline plotting the evolution of the clicks to NDC ratio for the top five operators. This allows affiliates to know which operators are providing better conversion rates and, since NDC is directly related to income from CPA smart deals, it enables affiliates to be at a better position to negotiate new deals.

In the case of operators, the first chart (revenue by position), is replaced by a bar chart plotting the traffic from affiliates, measured as click events for the top five affiliates and including the monthly evolution. The other two charts (clicks to NRC and clicks to NDC ratios respectively) are equally shown but referring to the top five affiliates partnered with the operator. This enables operators to identify which affiliates are sending traffic of higher quality; i.e., more easily translated into conversions.

Finally, as it could be seen in Fig. 1, a summary of information can be shown for any set of transactions, applying as many filters as the partner wants. Filters can also be set on custom attributes included in a transaction; therefore, enabling complex analytics. Blockverse allows partners to download this information as a CSV file that can be loaded into external business analytics tools.

\section{System Scalability}

Due to its nature, Blockverse is a system that must be designed with scalability in mind, since it needs to adapt to large amounts of 
incoming traffic (from all affiliates and operators subscribed in the platform). It is important that the system is responding at all times, properly recording all the transactions. Otherwise, lost transactions would not only mean a loss of trust in the analytics provided by the system but could also lead to potential losses of revenue for affiliates.

To prevent this from happening, the system is designed with a robust and reliable cloud-based architecture that facilitates scalability of the system as traffic increases.

The whole Blockverse system is deployed in Amazon Web Services. Fig. 4 summarizes the current cloud architecture.

AWs Cloud

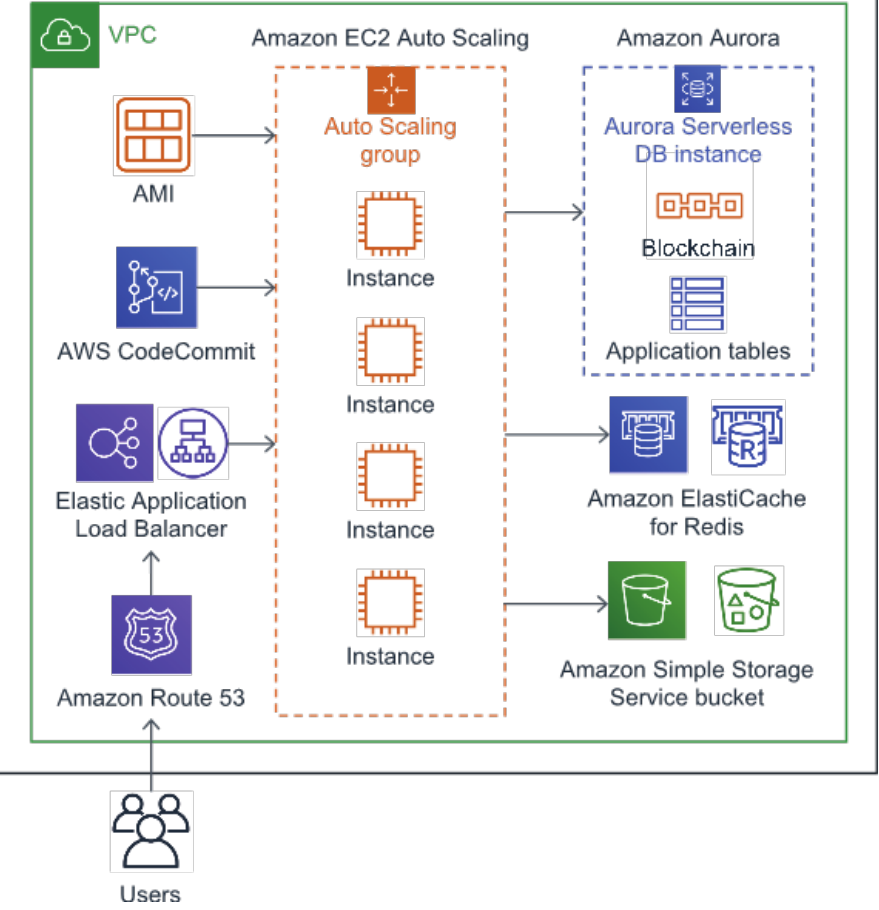

Fig. 4. Diagram of the cloud architecture of the Blockverse platform, representing how the different cloud services interact with each other.

The production environment is deployed within an autoscaling group, a mechanism that allows to provision new instances or destroy them based on traffic or CPU usage. Every time a new instance is provisioned, it launches the latest version of the Blockverse AMI (Amazon image) and checks out the most recent software release from CodeCommit (Amazon's source code repository) before starting to attend requests. Additionally, new software releases are rolled out to instances in the autoscaling group progressively, in order to prevent service interruption.

Data is stored in a relational database which is deployed in an Aurora serverless instance within Amazon RDS (relational database service). This database contains both the application tables and the blockchain itself. Aurora serverless enables automatic scalability based on traffic, provisioning additional compute units as traffic increases. Replication and automated backups are automatically managed by RDS.

Additionally, an in-memory Redis-based database is used for managing session information in Blockverse, using Amazon ElastiCache service. Also, S3 (simple storage service) buckets allow to store certain objects that are exchanged between partners, such as assets that they can upload to a shared library.

An application load balancer is in charge of redirecting traffic to the different instances in the autoscaling group. These two elements are configured together so that the load balancer is aware of which instances are available at each time. Finally, Route 53 is used to configure DNS entries for resolving domain names to the load balancer, and SSL certificates are also managed by Amazon to ensure secure communication between Blockverse and the users.

All the system is deployed within a VPC (virtual private cloud) and security groups have been configured to allow only authorized communications between the different services.

\section{CONCLUSIONS AND FutURE WORK}

In this paper we have presented Blockverse, a blockchain-based platform for tracking transactions in affiliate systems. At its current implementation, the system is deployed in the iGaming industry. In this industry, we can distinguish two different parties: operators (companies licensed to operate in the market) and affiliates, who redirect paid traffic to operators.

This industry is complex inasmuch as operators and affiliates can establish very complex agreements on how the former will pay the latter based on the received traffic. Generally, operators will pay affiliates based on actions (e.g., the user deposits some amount of money in the platform) or as a revenue share of the income generated by the user. However, the specifics of these agreements can be quite intricate, depending on the type of ad, the location of the banner, etc.

The Blockverse platform provides means for affiliates to record the traffic they generate to operators, and for operators to record the actions performed by users in their platform. Blockverse keeps track (using a mechanism conveniently called trackers) of how affiliate traffic converts into registrations, deposits or bets. Both partners can record custom fields associated to each transaction, that can later be used to perform advanced filtering and analytics.

Additionally, partners can use Blockverse to negotiate and establish smart deals, which rules determining the conditions in which affiliates will perceive money for traffic redirected to the operator's platform. Negotiation of deals has an established workflow that ensures that both partners agree on the final details. Trackers can be assigned to different deals, based on the different ways in which affiliates get traffic to operators, such as different money sites or positions.

The kind of industry in which Blockverse takes place imposes remarkable requirements when it comes to availability and scalability. For example, missing some API requests can have a direct impact in the revenue perceived by affiliates. To tackle this, we have designed a system which is entirely cloud-based, relying on different AWS tools to ensure that scalability is guaranteed, including serverless deployments of the database auto-scaling setups assisted by load balancers for the computing infrastructure.

In the shortcoming future, Blockverse functionality and its underlying architecture can be further improved. Regarding functionality, a broader consideration of CPA smart deals could be interesting besides NDC events. For example, register events could constitute another action which is a source of revenue for the affiliate, and which at this moment is not supported.

Regarding the Blockverse deployment on cloud, it could be interested to migrate the blockchain from its current SQL implementation to QLDB (Quantum Ledger Database), a recent serviced introduced by Amazon [14] that provides a quite natural mechanism to provide a transactions ledger mimicking the structure of a blockchain and without need to implement our own verification and integrity mechanisms.

These future improvements are already considered in the Blockverse roadmap as future development. 
[1] Amazon, "Amazon.com Associates Central," [Online]. Available: https:// affiliate-program.amazon.com/help/node/topic/GRXPHT8U84RAYDXZ. [Accessed 2003 2020].

[2] S. Nakamoto, "Bitcoin: A Peer-to-Peer Electronic Cash System," 2009. Available: https://bitcoin.org/bitcoin.pdf [Accessed 1206 2020].

[3] V. Buterin, "A next-generation smart contract and decentralized application platform," Ethereum White Paper. 2014. Available: https://ethereum.org/ whitepaper/ [Accessed 1206 2020].

[4] Z. Zheng, S. Xie, H.-N. Dai, X. Chen and H. Wang, "Blockchain challenges and opportunities: A survey," International Journal of Web and Grid Services, vol. 14, no. 4, p. 352-375, 2018.

[5] A. Baldominos and Y. Saez, "Coin.AI: A Proof-of-Useful-Work Scheme for Blockchain-Based Distributed Deep Learning," Entropy, vol. 21, no. 8, 2019.

[6] J. Blasingame, "Blockchain Isn't The End Of Trust, It's The Future Of Trust," Forbes, 07 Jul 2019.

[7] A. Ashiq, M. Sporny and A. Sill, "Blockchain Standards for Compliance and Trust," IEEE Cloud Computing, vol. 4, no. 4, p. 84-90, 2017.

[8] F. Hawlitschek, B. Notheisen and T. Teubner, "The limits of trust-free systems: A literature review on blockchain technology and trust in the sharing economy," Electronic Commerce Research and Applications, vol. 29 , p. 50-63, 2018.

[9] A. V. Ertemel, "Implications of Blockchain Technology on Marketing," Journal of International Trade, Logistics and Law, vol. 4, no. 2, p. 35-44, 2018.

[10] M. Kotila, R. C. Rumin, A. Phansalkar, J. Manner and M. Pärssinen, "Is Blockchain Ready to Revolutionize Online Advertising?," IEEE Access, vol. 6, p. 54884-54899, 2018.

[11] N. Kshetri and J. Voas, "Online Advertising Fraud," Computer, vol. 52, no. 1, p. 58-61, 2019.

[12] M. Weijer, "Providing Trust in Affiliate Marketing through Blockchain Technology," Utrecht University, 2019.

[13] M. J. Bordash, M. J. Hudson and C.-H. Wong, "Tracking transactions through a blockchain". USA Patent US20180053161A1, 2016.

[14] Amazon, "Amazon Quantum Ledger Database (QLDB)," [Online]. Available: https://aws.amazon.com/qldb/. [Accessed 0604 2020].

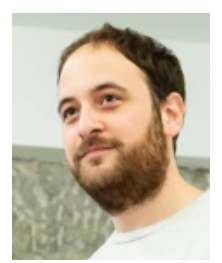

\section{A. Baldominos}

A. Baldominos received his Ph.D. in computer science and technology by Universidad Carlos III de Madrid, Spain, in 2018, with his dissertation focusing on the automatic evolutionary design of deep and convolutional neural networks. He works as a Research Assistant in Universidad Carlos III de Madrid (Spain) and teaches different courses in private colleges, such as Universidad Internacional de la Rioja and CUNEF Universidad. He is also founder and CTO at Blockverse Solutions. Dr. Baldominos is member of AEPIA, the Spanish Association for Artificial Intelligence, and is a former member of IEEE, ACM and AAAI.

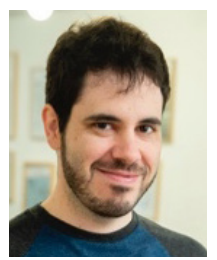

\section{J. L. López-Sánchez}

J.L. López-Sánchez received his B.Sc. in computer science by Universidad Carlos III de Madrid, Spain, in 2014, with a minor in artificial intelligence. He currently works as a software developer for the group Multimedia and Teaching Innovation at Universidad Carlos III de Madrid. He is also founder and $\mathrm{CIO}$ at Blockverse Solutions.

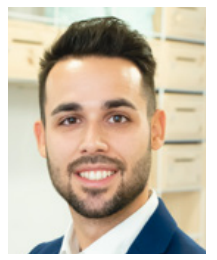

M. Acevedo-Aguilar

M. Acevedo-Aguilar received his B.Sc. in electronic engineering by Universidad Carlos III de Madrid, Spain, in 2012, focused on embedded software. He works as Project Manager and Product Owner at different startups. He is also an experienced BI Consultant as well as $\mathrm{CEO}$ and founder at Blockverse Solutions. 